\title{
Eumelanin Adsorption on Silicon: Optical Properties of Si(001)-adsorbed Eumelanin Tetrameric Protomolecules
}

Elena Molteni, ${ }^{* \dagger}$ Giancarlo Cappellini, ${ }^{\ddagger} \uparrow$ Roberto Cardia, ${ }^{\ddagger}$ Giovanni Onida, ${ }^{\dagger}$ and

$$
\text { Guido Mula }
$$

$\dagger$ †ipartimento di Fisica, Università degli Studi di Milano, Via Celoria 16, 20133 Milano, Italy, and European Theoretical Spectroscopy Facility (ETSF)

$\ddagger$ Dipartimento di Fisica, Università degli Studi di Cagliari, Cittadella Universitaria di Monserrato, S.P. Monserrato-Sestu, Km. 0.700,09042 Monserrato (CA), Italy ฯIstituto Officina dei Materiali CNR-IOM, Unità di Cagliari SLACS, Cittadella Universitaria di Monserrato, S.P. Monserrato-Sestu, Km. 0.700, 09042 Monserrato (CA), Italy

E-mail: elena.molteni@mi.infn.it Phone: +3902503 17377 


\begin{abstract}
We predict the optical properties - in particular optical absorbance and Reflectance Anisotropy Spectra (RAS) - of the silicon(001) surface organically functionalized by the adsorption of chosen tetrameric eumelanin protomolecules. These $\mathrm{Si}(001)$ :tetramer systems can be considered as models of hybrid (porous) silicon-eumelanin interfaces, a system with potential applications in photovoltaics. In spite of a weak effect of tetramer adsorption on $\mathrm{Si}(001)$ overall absorption spectra, first-principles results based on plane wave density functional theory allowed us to identify specific regions, in the interesting IR and visible range, where the adsorbed molecules yield large enhancements in surface absorbance, and the appearance of new RAS features.
\end{abstract}

\title{
Introduction
}

Eumelanin is a photoprotective pigment, widely present in living organisms: it is interesting both for its biological role and in view of possible applications, e.g. as a photoactive layer in hybrid solar cells. Porous silicon (PSi) - eumelanin interfaces are interesting systems for photovoltaic applications, due to (a) the large surface-to-volume ratio of the porous substrate, (b) the peculiar broadband UV-vis absorption spectrum of the pigment, and its biocompatibility and biodegradability. Indeed the ability of eumelanin functionalization to extend the absorption range of PSi towards longer wavelengths has been demonstrated. ${ }^{1,2}$

The basic building blocks of eumelanin are 5,6-dihydroxyindole (DHI) - like molecules. Although the detailed 3D structure of the pigment is not known, a wealth of experimental measurements suggest the presence of locally ordered $\pi$-stacked arrangements of relatively small oligomers (like tetramers or pentamers) of DHI-like molecules, ${ }^{3-5}$ and the role of stacking and oligomerization in the emergence of the broadband eumelanin spectrum is actively studied from the computational point of view (e.g. ${ }^{6,7}$ ).

In particular, it has been demonstrated that the DHI-like monomers have an absorbance spectrum localized mainly in the UV, both in their isolated ${ }^{8}$ and stacked ${ }^{6,7}$ form. Therefore 
monomers alone can not be responsible for the typical eumelanin broadband absorption from IR to visible and UV, which is so important to shield living organisms from light-induced damages.

Studies of the electronic properties and optical absorption spectra of monomeric constituents of eumelanin - namely DHI, DHICA (5,6-dihydroxyindole-2-carboxylic acid), IQ (indolequinone) and MQ (quinone-methide) - and of small chromophores in other biomolecules have been addressed by some of us using a systematic approach in the last years. In many of those cases the role of the single small-scale constituent turned out to be of fundamental importance. ${ }^{9-11}$ In the case of eumelanin, as mentioned above, the scenario is different, ${ }^{6,8}$ and oligomerization needs to be taken into account in order to properly describe the experimental absorption spectrum of the pigment. All these experimental and computational results point to small oligomers as the most probable units undergoing stacking in the eumelanin pigment.

Computational investigations have been reported on the energetics, adhesion properties, electronic properties and interface stability of simplified models of the PSi-eumelanin interface, consisting of tetramers of DHI-like molecules interacting with the Silicon(001) surface. ${ }^{12,13}$

Clearly, a theoretical understanding of the effects of eumelanin on the optical properties of the substrate, in addition to the above-mentioned issues, is of paramount importance to control and optimize the behaviour of these composite materials both in terms of stability and photovoltaic properties. First principles calculations on organically functionalized surfaces are able to yield valuable highlights in this respect (e.g. ${ }^{14,15}$ ), also in cases where the experimental approach cannot reach a sufficiently detailed information, being therefore a powerful and fundamental tool in this field.

In this work we investigate, by plane wave density functional theory (DFT), the absorption spectra and Reflection Anisotropy Spectra (RAS) of the DHI-like tetramer - functionalized $\operatorname{Si}(001)$ surface, focusing on the effect of molecule adsorption in terms of sensitivity 
to the type of adsorbed molecule, and to its adsorption configuration.

\section{Methods}

\section{Computational details}

All the present calculations are performed within plane wave (PW) DFT ${ }^{16,17}$ using the exchange-correlation functional in the form by Perdew, Burke, and Ernzerhof (GGA-PBE). ${ }^{18}$ To describe the ion-electron interaction we use norm-conserving pseudopotentials of the Trouiller-Martins-type generated via the FHI98PP ${ }^{19}$ code.

The plane wave approach is the natural choice for describing periodic systems such as 3D crystals or 2D crystal surfaces, allowing one to study extended - in principle infinite systems by using a (relatively) small unit cell, thus reducing the computational cost. Since the focus of this work is on the $\mathrm{Si}(001)$ surface - periodic along the 2 surface directions functionalized by the adsorption of eumelanin protomolecules, we take advantage of the PW approach.

We model the $\mathrm{Si}(001)$ surface with a periodically repeated asymmetric slab, using a supercell containing 8 Si layers plus a vacuum region (including adsorbed molecules) of $\sim 1.74$ nm, equivalent in thickness to 12 - 13 Si layers. Our supercell corresponds to an $(8 \times 4)$ in-plane surface periodicity, with an area which is 4 times that of the $(4 \times 2) \mathrm{Si}(001)$ reconstruction, hence containing 16 of the Si dimers which are typical of the clean $\mathrm{Si}(001)$ surface. Our choice allows to model the adsorption of the investigated tetrameric protomolecules roughly parallel to the surface. The Si bottom layer is saturated by hydrogen atoms. For ground state calculations we sample the surface Brillouin zone $($ SBZ) with a $(4 \times 4)$ shifted Monkhorst-Pack mesh, ${ }^{20}$ where all the k-points where shifted by $1 / 4$ of the BZ width along the two in-plane directions. The kinetic energy cutoff for the planewave expansion is set to 20 Ha.

We compute optical properties within the Independent Particle Random Phase Approx- 
imation (IP-RPA), ${ }^{21}$ i.e., by computing the macroscopic dielectric function of the supercell as a sum of individual valence $(v)$ to conduction $(c)$ direct transitions between Kohn-Sham eigenstates:

$$
\operatorname{Im} \varepsilon_{j j}(\omega)=\frac{16 \pi}{\omega^{2}} \sum_{v, c, k}\left|\left\langle\psi_{v k}\left|(\mathbf{v})_{j}\right| \psi_{c k}\right\rangle\right|^{2} \delta\left(\epsilon_{c k}-\epsilon_{v k}-\omega\right) .
$$

Here $\mathbf{v}$ is the velocity operator and $j=x, y, z$.

For all the investigated systems, i.e. $\mathrm{Si}(001)$ :tetramer systems and the clean $\mathrm{Si}(001)$ surface, we compute the absorbance of the slab model for light at normal incidence ${ }^{22-24}$ as:

$$
A_{j}(\omega)=\frac{\omega L}{c} \operatorname{Im}\left(\varepsilon_{j j}(\omega)\right)
$$

Here $j=\operatorname{par} 1, \operatorname{par} 2$ labels the light polarization, $L$ is the length of the simulation cell along the surface normal, and $c$ is the velocity of light. We remark that the $A_{j}$ is independent on the thickness of the vacuum region. In particular, we choose as par 1 axis the direction of $\mathrm{Si}$ dimers of the clean $\mathrm{Si}(001)$ surface, and as par 2 the orthogonal in-plane direction, i.e. the Si dimer axis.

The RAS spectrum is obtained as ${ }^{25,26}$

$$
\frac{\Delta R}{R}(\omega)=\frac{4 \omega L}{c} \operatorname{Im}\left\{\frac{\Delta \varepsilon(\omega)}{\varepsilon_{\mathrm{bulk}}(\omega)-1}\right\}
$$

where $\Delta \varepsilon=\varepsilon_{\text {par } 1}-\varepsilon_{\text {par } 2}$, and $\varepsilon_{\text {bulk }}$ is the dielectric function of bulk Si.

We obtain converged optical spectra in the range of interest by including empty states with energy $\epsilon_{c k}$ up to $\approx 8 \mathrm{eV}$ above the valence band maximum, and by sampling the SBZ on a $(4 \times 4)$ shifted Monkhorst-Pack mesh,${ }^{20}$ where all the k-points where shifted by $1 / 4$ of the $\mathrm{BZ}$ width along the two in-plane directions.

In all cases a lorentzian broadening of $0.2 \mathrm{eV}$ is added to the $\delta$-like contributions from Eq. 1.

For ground-state geometries and electronic structure we rely on Quantum ESPRESSO, ${ }^{27,28}$ 
a suite of open-source codes for electronic structure calculations based on density functional theory, plane wave expansion of the electronic wavefunctions and ionic pseudopotentials, while in all the optical properties calculations we use Yambo, ${ }^{29}$ a plane wave code which allows to calculate the optical response starting from the previously generated Kohn-Sham wave functions.

(a)

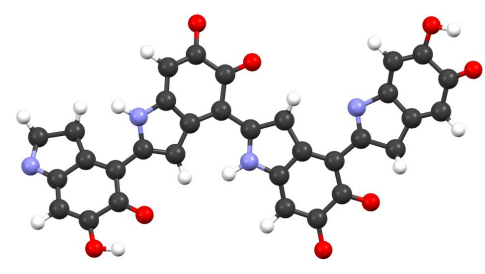

(c)

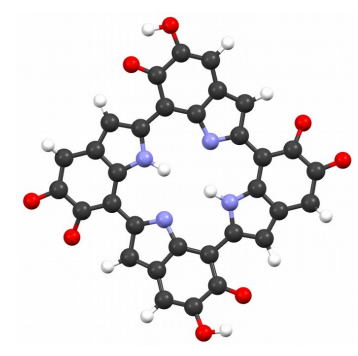

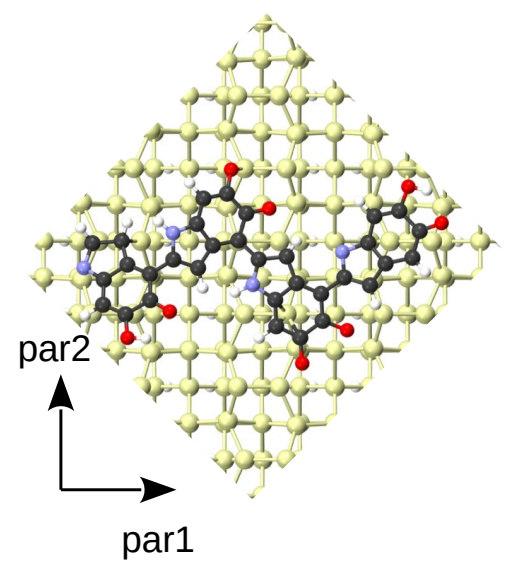

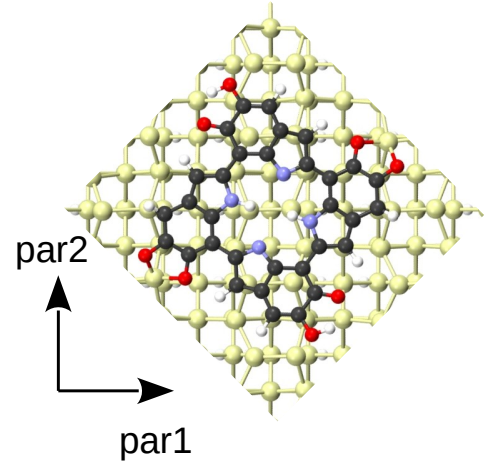

(b)

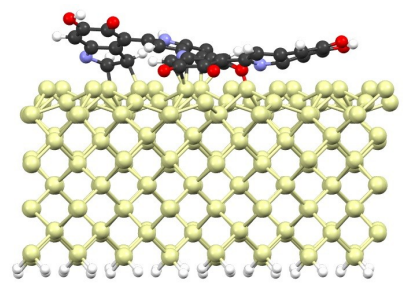

(d)

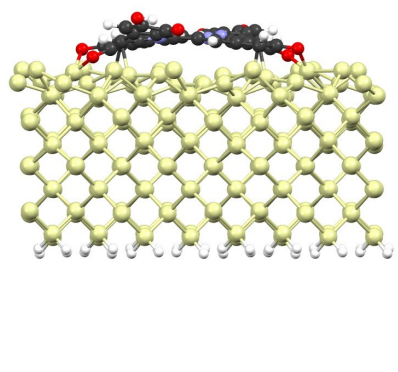

Figure 1: Geometries of: the PNZ tetramer in the gas phase (a) and adsorbed on the $\mathrm{Si}(001)$ surface in the $0^{\circ}$ orientation (b); the IMIM tetramer in the gas phase (c) and adsorbed on the $\mathrm{Si}(001)$ surface in the $0^{\circ}$ orientation $(\mathrm{d})$. Color codes: silicon atoms are depicted in pale yellow, carbon in black, nitrogen in indigo, oxygen in red, hydrogen in white. 


\section{Structural Details}

We consider adsorption of two different tetrameric eumelanin protomolecules, the "linear" PNZ one ${ }^{30}$ and the cyclic porphyrin-like IMIM one ${ }^{31}$ (Fig. 1), deposited on the clean $4 \times 2$ reconstructed $\mathrm{Si}(001)$ surface. Indeed, based on experimental data ${ }^{3-5}$ on the lateral size of stacked arrangements of protomolecules present in the eumelanin pigment, tetramers are among the most likely units undergoing stacking, and have therefore attracted interest in the literature.

Among the large variety of possible tetrameric protomolecules proposed in the literature (corresponding to different arrangements of four DHI-like monomers, their oxidation states etc), we chose the non-cyclic tetramer described by Panzella et al. ${ }^{30}$ (hereafter called PNZ) and the so-called IMIM porphyrin-like one ${ }^{31}$ as prototypes of two significantly different classes of tetramers. This allows us to investigate the dependence of the optical properties of the functionalized silicon surface on the type of adsorbed tetramer.

In order to sample the effect of variations in adsorption geometry on the optical properties of the functionalized silicon surface, we considered - for each of the two molecular species - three distinct adsorption configurations, only differing in the orientation of the tetramer with respect to the relevant directions on the $\mathrm{Si}(001)$ surface (the direction of silicon dimers and the one of dimer rows), while keeping the molecule roughly parallel to the Si surface in all cases. Based on the peculiar symmetry of the porphyrin-like IMIM tetramer, which is invariant (in its planar geometry and neglecting the protonation state of internal nitrogen atoms and external oxygen atoms) under 90 degrees rotations within the molecule plane, we considered, for both IMIM and PNZ, 22.5 degrees rotations, and restricted ourselves to three different orientations only, which we labeled as $0^{\circ}, 22.5^{\circ}, 45^{\circ}$, conventionally taking the first one as reference, as depicted in Figure 1. The relative differences in total energy among the investigated systems are in all cases very small (below $0.1 \%$ of the total energy of the adsorbed system), with the $0^{\circ}$ geometry being slightly energetically disfavored (favored, respectively) with respect to the other two orientations for PNZ (IMIM, respectively). 


\section{Results and discussion}

In Figure 2 we report Reflection Anisotropy spectra for the investigated $\mathrm{Si}(001)$ :X systems (where $\mathrm{X}$ is either the PNZ or the IMIM tetramer, in one of the chosen in-plane orientations, i.e. $\left.0^{\circ}, 22.5^{\circ}, 45^{\circ}\right)$, compared to the RAS spectrum of the clean $\operatorname{Si}(001)$ c $(4 \times 2)$ surface. The clean $\operatorname{Si}(001)$ RAS spectrum (cyan curve) displays two characteristic negative peaks, at $\approx 1.3$ - $1.4 \mathrm{eV}$ and at $\approx 3 \mathrm{eV}$, in agreement with previous experimental ${ }^{32}$ and computational $^{14,33-35}$ literature.
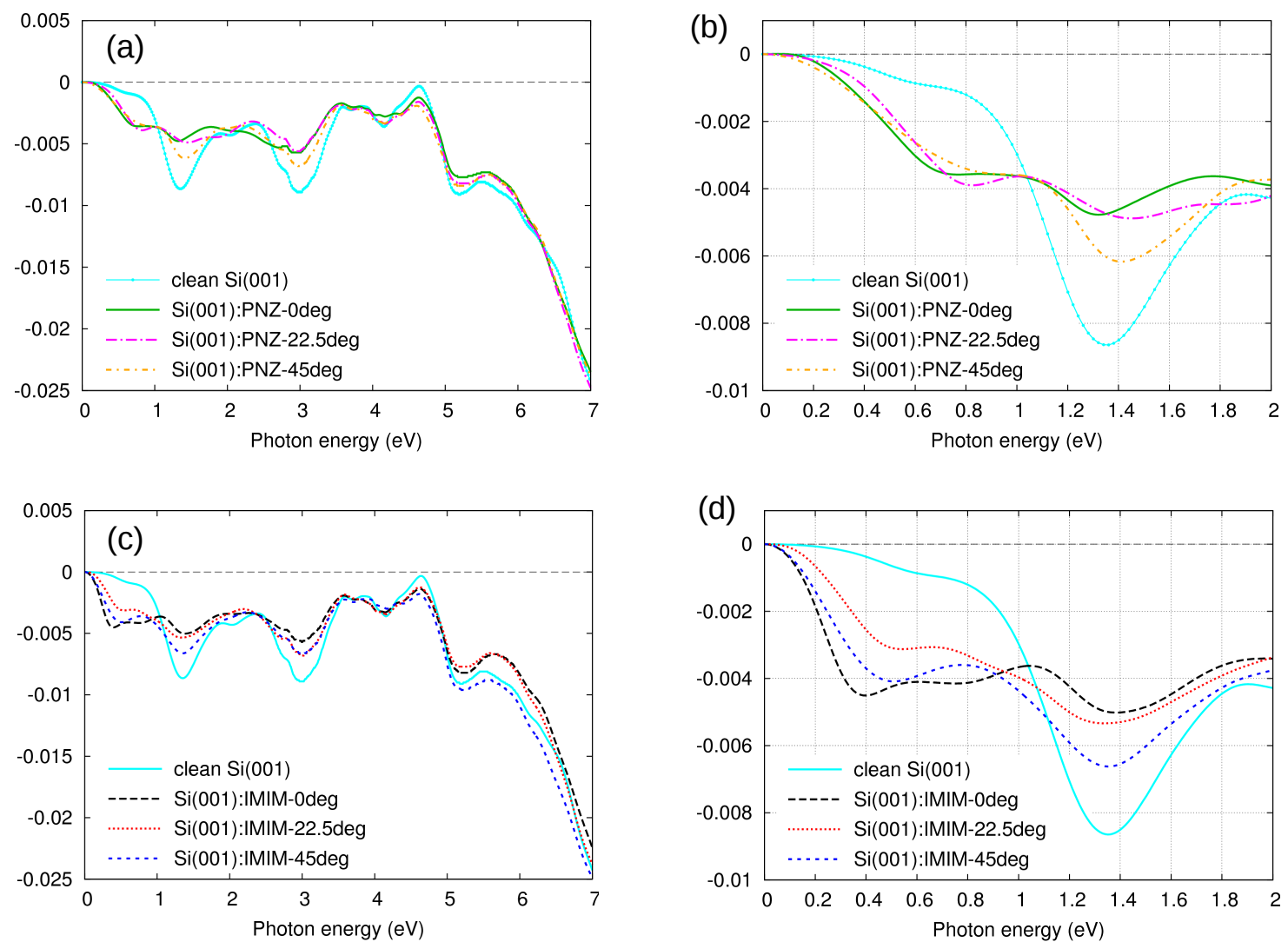

Figure 2: RAS spectra of: $\mathrm{Si}(001): \mathrm{PNZ}$ with 3 different orientations of the tetramer on the surface plane $\left(0^{\circ}\right.$ dark green solid curve, $22.5^{\circ}$ magenta long dashed-dotted curve, $45^{\circ}$ orange short dashed-dotted curve), compared with clean $\mathrm{Si}(001)$ (cyan solid curve with dots): (a) 0-7 eV range, (b) 0-2 eV range; $\mathrm{Si}(001)$ :IMIM ( $0^{\circ}$ black long dashed curve, $22.5^{\circ}$ red dots, $45^{\circ}$ blue short dashed curve), compared with clean $\mathrm{Si}(001)$ (cyan solid curve): (c) 0-7 eV range, (d) 0-2 eV range. 
The main changes induced by adsorption of the investigated tetrameric protomolecules, the "linear" PNZ and the cyclic porphyrin-like IMIM, in the chosen geometries, are a reduction in intensity of the two above-mentioned clean $\mathrm{Si}(001)$ RAS peaks, and the appearance of a further negative feature at lower energy $(<1 \mathrm{eV})$. The quenching of the two negative clean $\mathrm{Si}(001)$ peaks is here less dramatic than the one observed for the same surface, functionalized by the adsorption of uracil-like pyrimidinic nucleobases: ${ }^{14}$ this may be due to the fact that in the present $\mathrm{Si}(001)$ :tetramer systems the adsorbed molecules only partially saturate the silicon dangling bonds on the surface, leaving a much higher density of unsaturated silicon surface dangling bonds with respect to the case of, ${ }^{14}$ as discussed in the Supporting Information. The new feature below $1 \mathrm{eV}$ appearing in RAS spectra of $\mathrm{Si}(001)$ :tetramer systems is sensitive, both in intensity and in energy position, to the type of adsorbed molecule and, in the IMIM case, also to its adsorption geometry (see figure).

RAS features, and their changes upon molecule adsorption, can be related to absorption spectra for light polarized along the two corresponding in-plane directions, par 1 and par2, as can be observed in Figure 3. Optical absorbance spectra of the clean $\operatorname{Si}(001)(4 \times 2)$ surface display the well-known typical peak at $\approx 1.3 \mathrm{eV}$, with a shoulder at $\approx 3 \mathrm{eV}$ (not shown), for light polarization along the direction of Si dimer rows, as visible in the right panel of Fig. 3. These are the two features originating the two negative peaks in the absorption anisotropy $\Delta \mathrm{A}(\omega)=\mathrm{A}_{\text {par } 1}(\omega)-\mathrm{A}_{\text {par } 2}(\omega)$ of the clean surface (cyan curves in Fig.4), corresponding to the two well-known clean $\mathrm{Si}(001)$ RAS negative peaks.

In tetramer-functionalized systems the two absorption features at $\approx 1.3 \mathrm{eV}$ (right panels of Fig 3) and $\approx 3 \mathrm{eV}$ (not shown) for polarization along $\mathrm{Si}$ dimer rows are quite reduced in intensity, without being completely quenched. The same holds for the corresponding negative absorption anisotropy peaks (Fig 4). Accordingly, the negative RAS peaks at the same frequencies are still present, although with reduced intensity (Fig. 2).

On the other hand, as shown in the right panel of Fig. 3, absorption spectra for light polarized along Si dimer rows (par2 direction) show a rather large increase in intensity below 
(a)

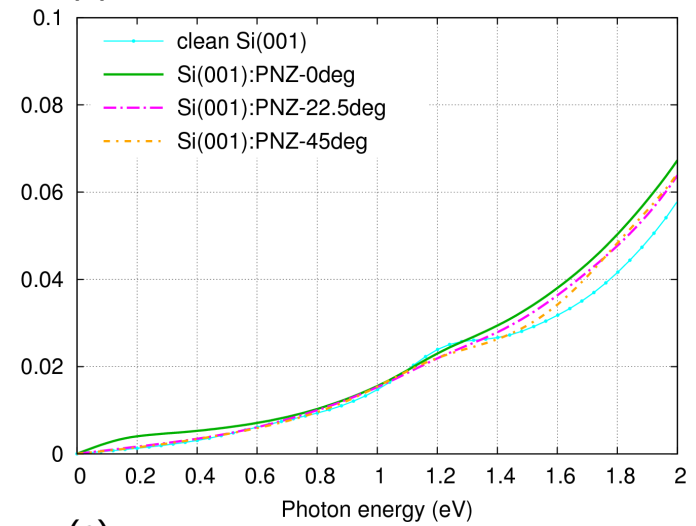

(c)

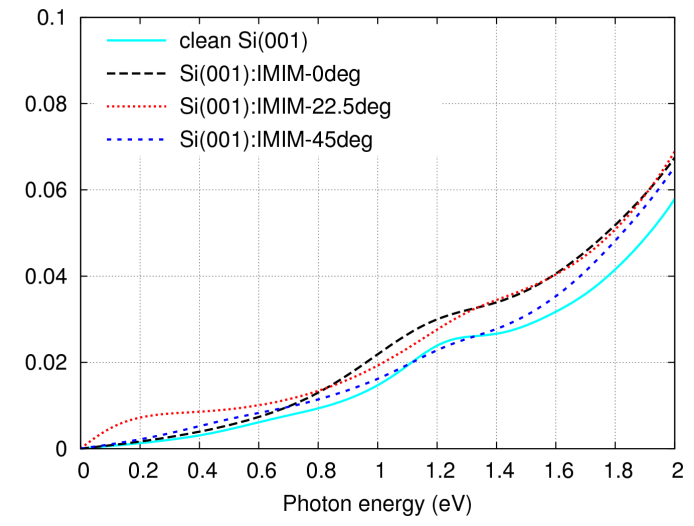

(b)

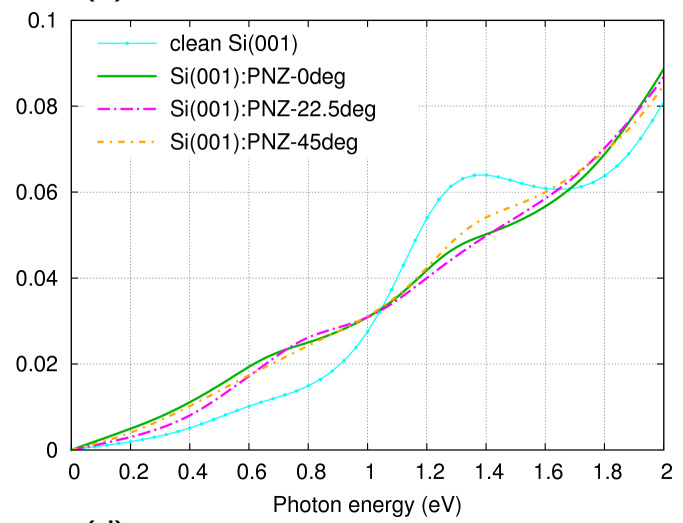

(d)

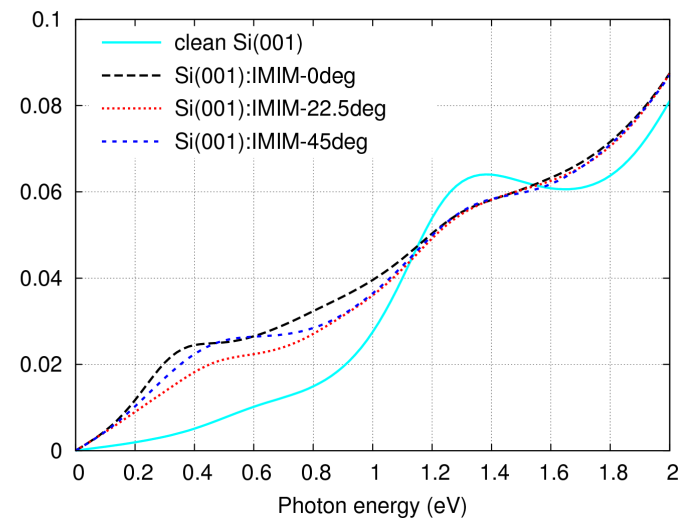

Figure 3: Optical absorbance $\mathrm{A}(\omega)$ of: $\mathrm{Si}(001)$ :PNZ systems $\left(0^{\circ}, 22.5^{\circ}, 45^{\circ}\right)$ and clean $\mathrm{Si}(001)$ for light polarized along the par1 (a) and $\operatorname{par} 2$ (b) directions; $\mathrm{Si}(001)$ :IMIM systems $\left(0^{\circ}\right.$, $\left.22.5^{\circ}, 45^{\circ}\right)$ and clean $\mathrm{Si}(001)$ for light polarized along the par 1 (c) and par2 (d) directions. Same color codes as in Fig. 2. par 1 and par 2 are the two mutually orthogonal directions, both parallel to the surface plane, used for RAS calculations. 

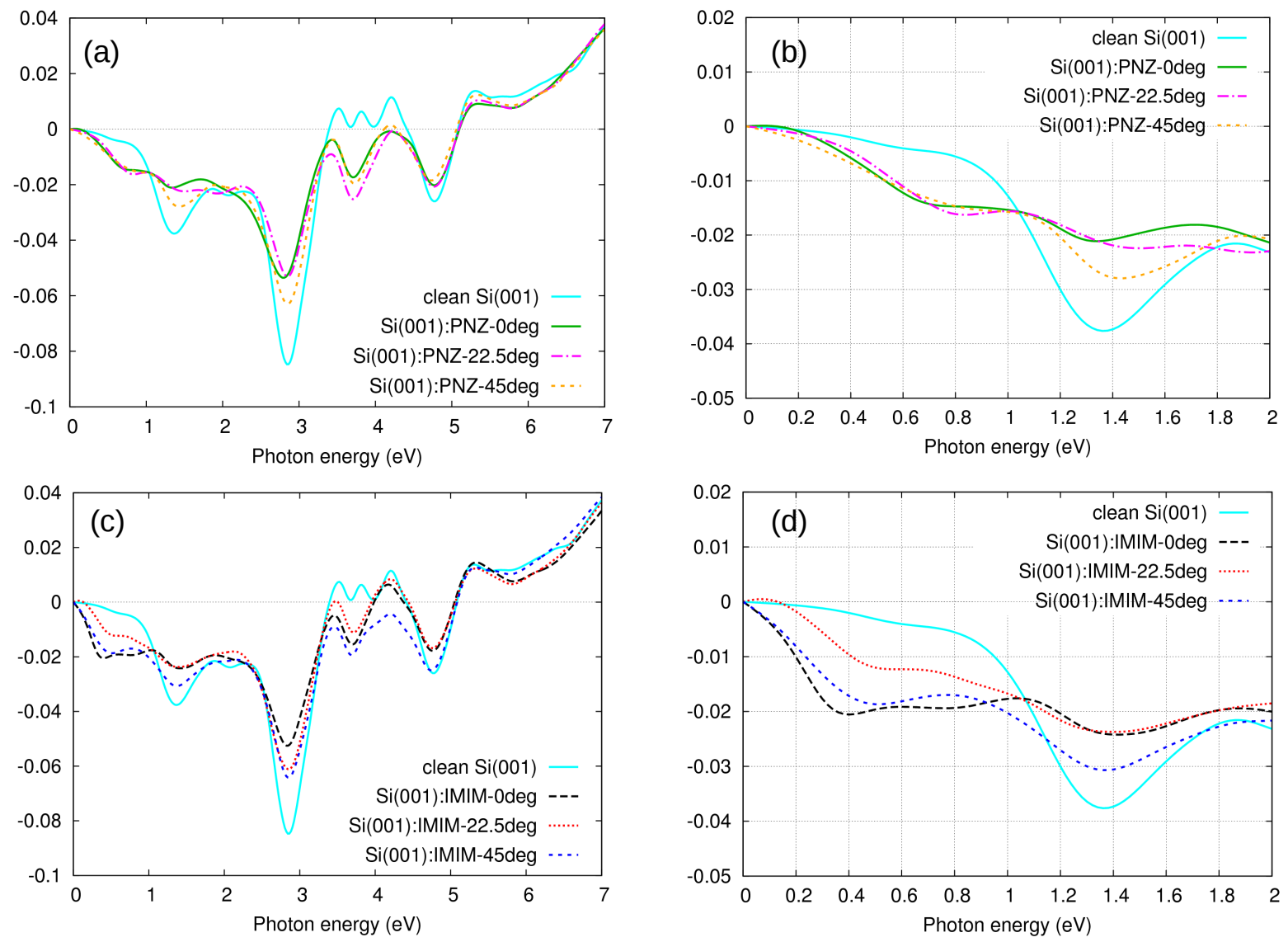

Figure 4: Optical absorbance anisotropy, $\Delta A(\omega)=A_{\text {par1 } 1}(\omega)-A_{\text {par2 }}(\omega)$, for: $\operatorname{Si}(001)$ :PNZ $\left(0^{\circ}\right.$, $\left.22.5^{\circ}, 45^{\circ}\right)$, compared with clean $\mathrm{Si}(001)$ : (a) 0-7 eV range, (b) 0-2 eV range; $\mathrm{Si}(001)$ :IMIM $\left(0^{\circ}, 22.5^{\circ}, 45^{\circ}\right)$, compared with clean $\mathrm{Si}(001)$ : (c) 0-7 eV range, (d) 0-2 eV range. Same color codes as in Fig 2. 
$1 \mathrm{eV}$ upon tetramer functionalization. Such enhancement is either low or absent for the par 1 polarization (left panel of the same Figure). The absorption anisotropy spectrum becomes accordingly more negative upon molecule adsorption in the region below $1 \mathrm{eV}$ (see right panels of Fig. 4), as well as the RAS spectrum (Fig. 2).

Overall, silicon(001) surface functionalization by the monolayer/submonolayer adsorption of the investigated tetrameric eumelanin protomolecules in the chosen geometries induces some change in the optical absorption spectra for in-plane polarizations (Fig. 5). Such changes are only sizable in the lower energy part (0-2 eV), where calculated spectra allow one to predict measurable effects.

Indeed the integrated absorption change for in-plane polarization in the tetramer-functionalized $\mathrm{Si}(001)$ surface with respect to the clean one ranges from $+1.6 \%$ to $+2.15 \%$ if referred to the whole investigated energy interval, i.e. $0-7 \mathrm{eV}$ (from $+1.6 \%$ to $+1.7 \%$ for $\mathrm{Si}(001)$ :PNZ systems, from $+1.9 \%$ to $+2.15 \%$ for $\operatorname{Si}(001)$ :IMIM systems, considering for each the three analyzed orientations: $\left.0^{\circ}, 22.5^{\circ}, 45^{\circ}\right)$. By restricting the integration interval to the low energy range up to the upper visible limit $(3.1 \mathrm{eV})$, the integrated absorption variation of $\mathrm{Si}(001)$ :X systems with respect to clean $\mathrm{Si}(001)$ ranges from $-0.9 \%$ to $+4.3 \%$ (from $-0.9 \%$ to $+1 \%$ for $\operatorname{Si}(001)$ :PNZ systems, from $+2.8 \%$ to $4.3 \%$ for $\operatorname{Si}(001)$ :IMIM ones).

By focusing on specific frequencies, within the IR and visible region, we find in some cases substantial changes of absorbance upon surface functionalization, and different trends for the $\mathrm{Si}(001): P N Z$ vs. $\mathrm{Si}(001): I M I M$ systems. In $\mathrm{Si}(001): P N Z$ systems, for instance, moving from the low energy end of the spectrum up to $2 \mathrm{eV}$, regions where tetramer adsorption enhances surface absorbance alternate with other ones where the opposite effect is observed. More in detail, let us consider the three investigated $\mathrm{Si}(001)$ :PNZ geometries $\left(0^{\circ}, 22.5^{\circ}, 45^{\circ}\right)$ : at $0.8 \mathrm{eV}$ their absorbance enhancement with respect to the clean $\mathrm{Si}(001)$ surface ranges from $+40 \%$ to $+49 \%$ of the corresponding clean $\mathrm{Si}(001)$ absorbance; at $1.3 \mathrm{eV}$, on the contrary, they undergo a reduction in absorbance ranging from $-17 \%$ to $-21 \%$; at $1.8 \mathrm{eV}$ the variation is positive again, ranging from $+12 \%$ to $+13 \%$. In $\mathrm{Si}(001)$ :IMIM systems, on the other 
(a)

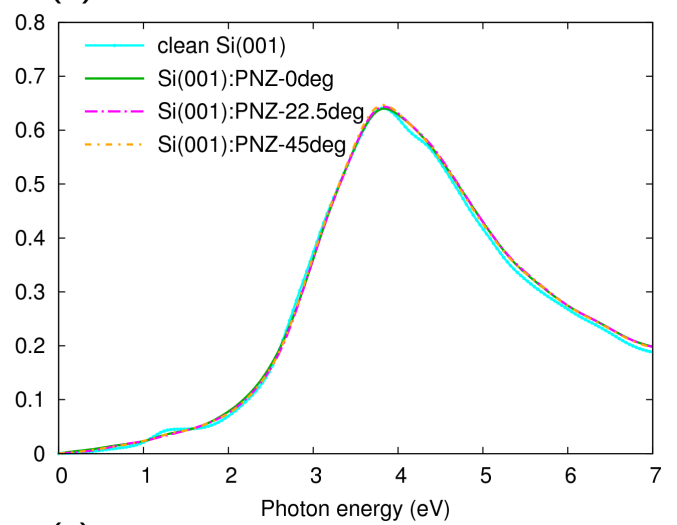

(c)

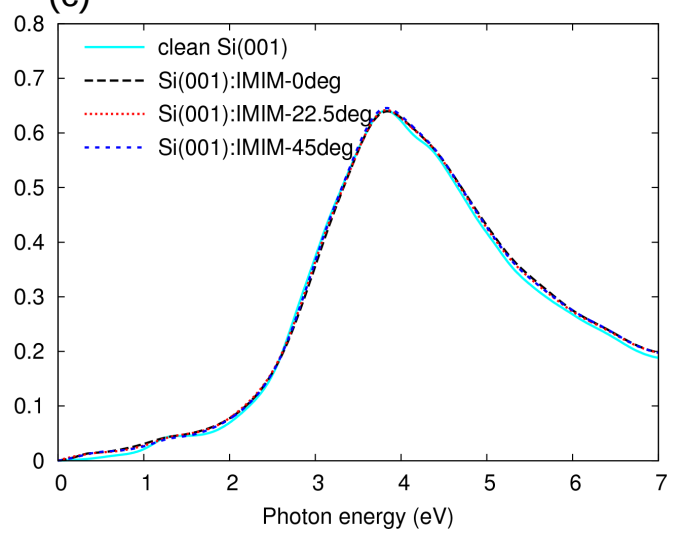

(b)

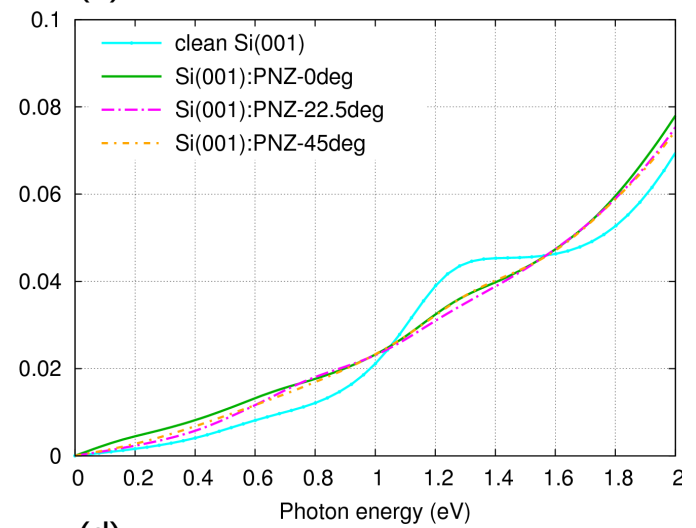

(d)

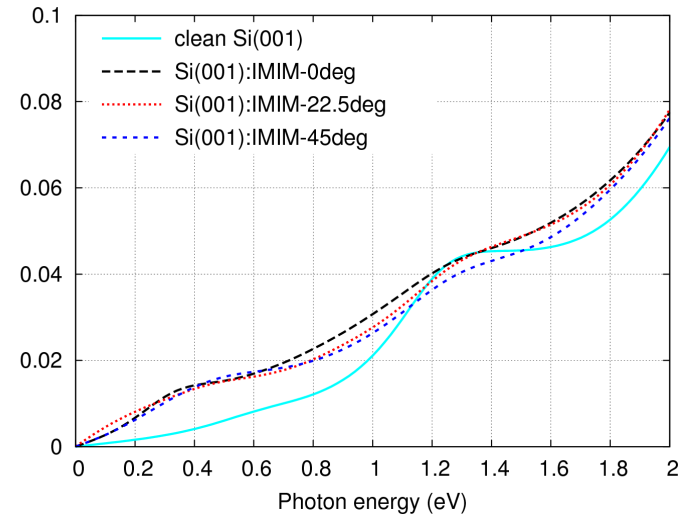

Figure 5: Optical absorbance $\mathrm{A}(\omega)$, averaged on the two mutually orthogonal in-plane polarization directions, par 1 , par 2 , for the the $\operatorname{Si}(001)$ : PNZ systems $\left(0^{\circ}, 22.5^{\circ}, 45^{\circ}\right)$, compared to clean $\operatorname{Si}(001)(\mathrm{a}),(\mathrm{b})$, and for the $\operatorname{Si}(001)$ :IMIM systems $\left(0^{\circ}, 22.5^{\circ}, 45^{\circ}\right)$, compared to clean $\operatorname{Si}(001)$ (c), (d). Same color codes as in Fig 2. 
hand, the absorbance variation induced by molecule adsorption is positive in almost all cases throughout the $0-2 \mathrm{eV}$ interval. In particular, at $0.4 \mathrm{eV}$ their absorbance is enhanced by $+227 \%$ to $+247 \%$ of the corresponding clean $\mathrm{Si}(001)$ absorbance, and at $1.8 \mathrm{eV}$ the enhancement ranges from $+13 \%$ to $+17 \%$. Specific theoretical energy values may be subject to the well-known error due to Kohn-Sham DFT gap underestimation, and may hence be shifted in reality. The use of hybrid exchange-correlation functionals such as the B3LYP one may improve the results, mainly by an enlargement of the energy gap between filled and empty electronic states; however this should not affect the general trends observed here, nor the important finding regarding the possibility of selectively enhancing the absorbance of the $\mathrm{Si}(001)$ surface at specific frequencies through eumelanin functionalization, as discussed below.

The adsorption of several layers of eumelanin tetramers - beyond the scope of this work - may be expected to have larger effects on the optical properties of the silicon surface.

These results regarding the "selective" optical response (i.e. absorption at specific frequencies) and the overall one (integrated absorption) of eumelanin-functionalized $\mathrm{Si}(001)$ could be of great importance for applicative scopes, mainly to boost the harvesting of photons in silicon-made devices either in selected energy slots or in extended energy ranges. The main message emerging from the present results is that to enhance the overall/wide-range absorbance of silicon surfaces one should probably consider several layers of adsorbed eumelanin, while a single eumelanin layer should be sufficient for strong selective absorbance enhancement at specific frequencies.

In order to identify the role of eumelanin tetramers and of the substrate in the predicted absorption (and absorption anisotropy and RAS) changes, we analyzed the spatial localization of the occupied and empty states involved in dipole-allowed transitions. To this aim, we modified Eq. 1 by weighting valence and conduction states according to their localization $W_{v k}^{\Phi}=\sum_{\phi \in \Phi}\left|\left\langle\phi \mid \psi_{v k}\right\rangle\right|^{2}$ and $W_{c k}^{\Phi}=\sum_{\phi \in \Phi}\left|\left\langle\phi \mid \psi_{c k}\right\rangle\right|^{2}$, respectively, where $\Phi$ indicates either the set of $\mathrm{Si}$ (substrate) or tetramer (molecule) atoms, and $\phi$ an atomic orbital belonging to one 

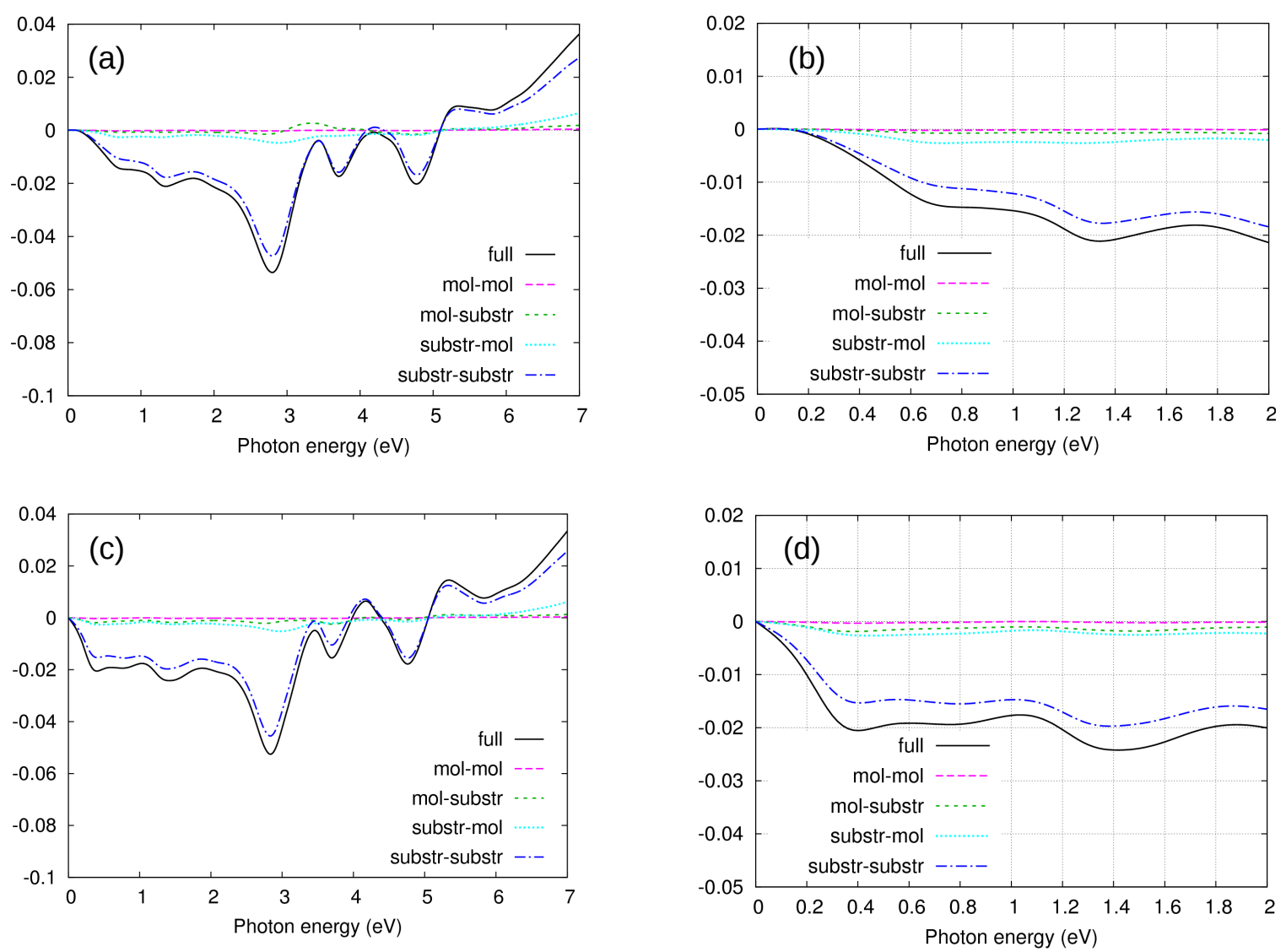

Figure 6: Optical absorbance anisotropy $\Delta \mathrm{A}(\omega)=\operatorname{Apar} 1(\omega)$ - Apar2( $\omega)$ (black solid curve) and its decomposition in terms of contributions (other colors) due to transitions from valence to conduction states mostly localized on the substrate or on the tetramer molecule, for the $\mathrm{Si}(001): \mathrm{PNZ}-0^{\circ}$ system (a,b), and for the Si(001):IMIM-0 system (c,d). 
of these two parts: ${ }^{36}$

$$
\operatorname{Im} \varepsilon_{j j}^{\Phi \Phi^{\prime}}(\omega)=\frac{16 \pi}{\omega^{2}} \sum_{v, c, k} W_{v k}^{\Phi} W_{c k}^{\Phi^{\prime}}\left|\left\langle\psi_{v k}\left|(\mathbf{v})_{j}\right| \psi_{c k}\right\rangle\right|^{2} \delta\left(\epsilon_{c k}-\epsilon_{v k}-\omega\right) .
$$

Through this procedure we can disentangle four different contributions in optical spectra, according to the possible choices of $\Phi$ and $\Phi^{\prime}$.

By thus decomposing the in-plane absorption anisotropy $\Delta \mathrm{A}(\omega)$ (more sensitive to surface details with respect to total absorption) in terms of contributions coming from valence to conduction transitions involving states mostly localized on the silicon substrate, or on the adsorbed tetramer (as shown in Fig. 6 for the $\mathrm{Si}(001): \mathrm{PNZ}-0^{\circ}$ and $\mathrm{Si}(001): \mathrm{IMIM}-0^{\circ}$ cases), we found that 1$)$ the largest contribution in the investigated energy range (0-7 eV) always stems from substrate-substrate transitions (blue dashed-dotted curves in the Figure), 2) we can not identify specific energy regions where the relative contribution from moleculemolecule transitions (magenta long dashed curves) is appreciably higher than in the rest of the spectrum.

By looking in more detail at these decompositions, we can identify differences among the various $\mathrm{Si}(001)$ :tetramer systems in the detailed behaviour of the molecule-molecule contribution to total absorption and to absorption anisotropy. As an example Fig. 7 shows this contribution to absorbance for the two in-plane polarizations, par1 and par2, for the $\mathrm{Si}(001): \mathrm{IMIM}-0^{\circ}$ and the $\mathrm{Si}(001): \mathrm{IMIM}-45^{\circ}$ cases. In the $\mathrm{Si}(001): \mathrm{IMIM}-45^{\circ}$ system (right panel of the Figure) we can observe a remarkable anisotropy between the two chosen inplane polarization directions, par1 and par2, at energies below $2 \mathrm{eV}$, which is absent in other cases (see e.g. the $\mathrm{Si}(001)$ :IMIM-0 ${ }^{\circ}$ system, left panel of the Figure).

This low energy par1-par2 anisotropy in the molecule-molecule contribution to absorbance of the full system (molecule + substrate) in the $\mathrm{Si}(001)$ :IMIM-45 case might be at least partially related to the relative orientation, in this specific system, of the chosen polarization directions with respect to the symmetry axes of the molecule. 
(a)

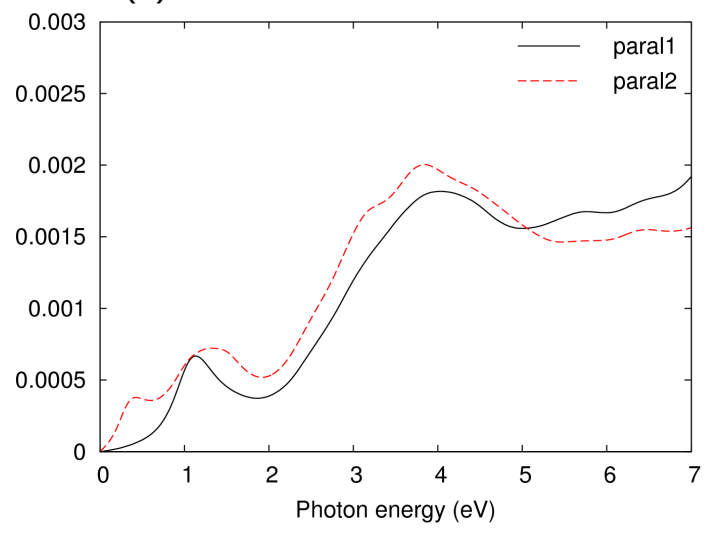

(b)

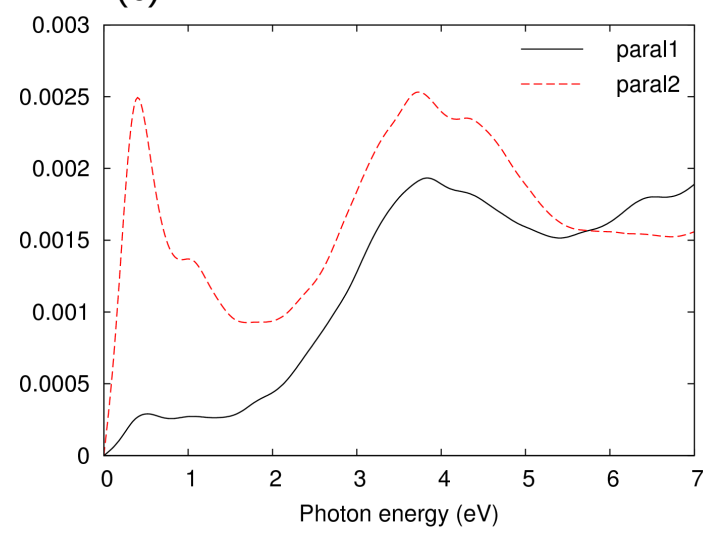

Figure 7: Molecule-molecule transitions contributions to the absorbance $A(\omega)$, for light polarization along the 2 in-plane directions par 1 (black solid curve), par2 (red dashed curve), for the following systems: (a) $\operatorname{Si}(001): I M I M-0^{\circ}$, (b) $\operatorname{Si}(001)$ :IMIM- $45^{\circ}$. 
In order to focus on effects and trends due to the molecule only, in Figure S1 of the Supporting Information we show the photoabsorption cross-section for chosen polarization directions of "IMIM-only-0deg-adsgeom" and "IMIM-only-45deg-adsgeom" models, i.e. of the gas phase IMIM molecule, frozen in its adsorbed geometry extracted respectively from the $\mathrm{Si}(001)$ :IMIM-0 $0^{\circ}$ and from the $\mathrm{Si}(001): \mathrm{IMIM}-45^{\circ}$ system. The $\mathrm{Y}$ and Z polarization directions used there for the IMIM-only-45deg-adsgeom model are rotated by $45^{\circ}$ with respect to par1 and par2 respectively, so that they would coincide with the par1 and par2 directions used for IMIM-only-0deg-adsgeom, if the two gas phase systems had an identical geometry. Indeed the photoabsorption cross-section spectrum of the IMIM-only-45deg-adsgeom model for light polarization along $\mathrm{Y}(\mathrm{Z})$ is similar - although not identical - to the one of the IMIM-only0deg-adsgeom model for par1 (par2) polarization.

The observed differences between the photoabsorption cross-section along Y (Z) of IMIMonly-45deg-adsgeom and that along par1 (par2) of IMIM-only-0deg-adsgeom can be understood if we keep in mind that the various "IMIM-only" models cannot be obtained from one another by simply rotating the IMIM molecule by the chosen angle within the molecular plane. Indeed, upon adsorption on the silicon surface, the tetramer molecule can (and does) undergo geometric rearrangements consisting, for the investigated systems, in distortions with respect to planarity and, in some cases, in a slight rotation. These geometric changes are in general dependent on the adsorption orientation.

Apart from the above-discussed differences in the details of the molecule-molecule contribution among the investigated models, in all cases the contribution to total absorbance and to in-plane absorbance anisotropy from molecule-molecule transitions is negligible (notice the different scales for absorbance between the plots in Fig. 7 and those in the left panels of Fig. 5).

Some of us had previously reported on the optical properties of the $\mathrm{Si}(001)$ surface functionalized by the adsorption of pyrimidinic nucleobases: ${ }^{14}$ in that case, RAS and absorbance anisotropy spectra were displaying regions where the contribution from molecule- 
molecule transitions was sizable (of the order of $40 \%$ for absorption anisotropy). In such regions, the spectra were quite sensitive to the type and geometry of the adsorbed molecule. Instead, regions where the contribution from substrate-substrate transitions was largely prevalent showed almost no dependence on molecule type and geometry. In the present $\mathrm{Si}(001)$ :tetramer systems we can not identify specific RAS regions which are much more sensitive to adsorbed molecule type and geometry than the rest of the spectrum; a moderate sensitivity to molecule orientation is present in the $\approx 1.2-3.2 \mathrm{eV}$ range for $\mathrm{Si}(001)$ :PNZ systems, and in the $0-1.8 \mathrm{eV}$ and 2.5-3.5 eV ranges and above $5 \mathrm{eV}$ for $\mathrm{Si}(001)$ :IMIM systems.

\section{Conclusions}

In this work we studied the optical properties of chosen eumelanin tetrameric protomolecules adsorbed on the $\operatorname{Si}(001)$ surface. These $\operatorname{Si}(001)$ :tetramer systems can be considered as simplified models of the porous silicon - eumelanin hybrid interface, which is currently studied in the literature in view of its possible applications in photovoltaics. Using a plane wave density functional theory approach, we calculated absorption and reflectance anisotropy spectra for two different tetramers, the non-cyclic PNZ one and the cyclic, porphyrin-like IMIM one, as prototypes of two significantly different classes of tetramers adsorbed on the $\mathrm{Si}(001)$ surface. For each of the two tetrameric species, we considered three different orientations on the silicon surface, while keeping the molecule roughly parallel to the surface in all cases. We were thus able to investigate the effect of molecule adsorption on the surface optical properties, and their sensitivity to molecule type and geometry. While total absorption is the more directly relevant property in view of photovoltaic applications, our choice of also computing RAS was motivated by its known sensitivity to surface details, which renders it particularly well-suited for an analysis of relatively small, although non-negligible, effects, such as those induced by monolayer/submonolayer adsorption of the chosen tetramers on $\mathrm{Si}(001)$.

The typical negative RAS peaks (at $\approx 1.3 \mathrm{eV}$ and $\approx 3 \mathrm{eV}$ ) of clean $\mathrm{Si}(001)$ are only 
partially quenched in the eumelanin-functionalized surface, while a new negative RAS feature appears below $1 \mathrm{eV}$, sensitive both to tetramer type and adsorption geometry. In optical absorption spectra, although the overall effect of adsorbed tetramers is weak, we can identify - in the relevant IR and visible range - spectral regions where surface functionalization induces non-negligible enhancements or decreases in absorbance with respect to the clean Si(001) surface. These absorbance variations are different for the two investigated molecular species, and in some cases large absorbance enhancements are observed, already within these monolayer / submonolayer model systems. This opens interesting perspectives, considering that the adsorption of more than one layer of eumelanin tetramers may be expected to affect the optical properties to an even larger extent. An investigation of the adsorption of stacked layers of eumelanin tetramers on $\mathrm{Si}(001)$ and its effects on the optical properties of the surface may be the subject of future works, taking advantage of the same plane wave approach used here, which allows such an extension by simply enlarging the cell size along the out-of-plane direction, while leaving it unchanged in the in-plane directions.

\section{Supporting Information Available}

Photoabsorption cross-section spectra for chosen polarization directions, for the gas phase IMIM molecule, frozen in its $\mathrm{Si}(001)$-adsorbed geometries.

\section{Acknowledgement}

E.M. acknowledges financial support from Regione Autonoma della Sardegna under Project R.A.S. L. R. 7/2007 CRP 78744 "Energy applications with porous silicon (EnAPSi)". G.C. acknowledges financial support from IDEA-AISBL, Bruxelles, Belgium. The authors acknowledge the CINECA award under the ISCRA initiative, for the availability of highperformance computing resources and support (project HP10CLJK67 "EuTetraS: Eumelanin tetrameric protomolecules adsorbed on the $\mathrm{Si}(001)$ surface"). 


\section{References}

(1) Pinna, E.; Melis, C.; Antidormi, A.; Cardia, R.; Sechi, E.; Cappellini, G.; d'Ischia, M.; Colombo, L.; Mula, G. Deciphering Molecular Mechanisms of Interface Buildup and Stability in Porous Si/Eumelanin Hybrids. Int. J. Mol. Sci. 2017, 18, 1567.

(2) Mula, G.; Manca, L.; Setzu, S.; Pezzella, A. Photovoltaic properties of PSi impregnated with eumelanin. Nanoscale Research Letters 2012, 7, 377.

(3) Cheng, J. I. N.; Moss, S. C.; Eisner, M.; Zschack, P. X-Ray Characterization of Melanins-I. Pigm. Cell Res. 1994, 7, 255.

(4) Cheng, J. I. N.; Moss, S. C.; Eisner, M.; Zschack, P. X-Ray Characterization of Melanins-II. Pigm. Cell Res. 1994, 7, 263.

(5) Zajac, G. W.; Gallas, J. M.; Cheng, J.; Eisner, M.; Moss, S. C.; AlvaradoSwaisgood, A. E. The fundamental unit of synthetic melanin: a verification by tunneling microscopy of X-ray scattering results. Biochim. Biophys. Acta 1994, 1199, 271-278.

(6) Molteni, E.; Cappellini, G.; Onida, G.; Mula, G. Extensive stacking of DHI-like monomers as a model of out-of-plane complexity in eumelanin protomolecules: Chemical and structural sensitivity of optical absorption spectra. Chem. Phys. 2019, 524, 92-100.

(7) Chen, C.-T.; Chuang, C.; Cao, J.; Ball, V.; Ruch, D.; Buehler, M. J. Excitonic effects from geometric order and disorder explain broadband optical absorption in eumelanin. Nature Commun. 2014, 5, 3859.

(8) Cardia, R.; Cappellini, G.; Pinna, E.; Tiddia, M. V.; Mula, G. Optical and Electronic Properties of Monomers of Eumelanin: A DFT and TD-DFT Computational Study. Optics and Photonics Journal 2016, 6, 41-47. 
(9) Stagi, L.; Chiriu, D.; Scholz, M.; Carbonaro, C. M.; Corpino, R.; Porcheddu, A.; Rajamaki, S.; Cappellini, G.; Cardia, R.; Ricci, P. C. Vibrational and optical characterization of s-triazine derivatives. Spectrochimica Acta Part A: Molecular and Biomolecular Spectroscopy 2017, 183, 348-355.

(10) Kumar, A.; Cardia, R.; Cappellini, G. Electronic and optical properties of chromophores from bacterial cellulose. Cellulose 2018, 25, 2191-2203.

(11) Kumar, A.; Cappellini, G.; Delogu, F. Electronic and optical properties of chromophores from hexeneuronic acids. Cellulose 2019, 26, 1489-1501.

(12) Antidormi, A.; Melis, C.; Canadell, E.; Colombo, L. Assessing the Performance of Eumelanin/Si Interface for Photovoltaic Applications. J. Phys. Chem. C 2017, 121, 11576-11584.

(13) Antidormi, A.; Aprile, G.; Cappellini, G.; Cara, E.; Cardia, R.; Colombo, L.; Farris, R.; d'Ischia, M.; Mehrabanian, M.; Melis, C. et al. Physical and Chemical Control of Interface Stability in Porous Si-Eumelanin Hybrids. J. Phys. Chem. C 2018, 122, $28405-28415$.

(14) Molteni, E.; Cappellini, G.; Onida, G.; Fratesi, G. Optical properties of organically functionalized silicon surfaces: Uracil-like nucleobases on $\operatorname{Si}(001)$. Phys. Rev. B 2017, 95,075437 .

(15) Molteni, E.; Fratesi, G.; Cappellini, G.; Onida, G. Optical Properties of Free and Si(001)-Adsorbed Pyrimidinic Nucleobases. Phys. Status Solidi B 2017, 1700497.

(16) Hohenberg, P.; Kohn, W. Inhomogeneous electron gas. Phys. Rev. 1964, 136, B864B871.

(17) Kohn, W.; Sham, L. J. Self-Consistent Equations Including Exchange and Correlation Effects. Phys. Rev. 1965, 140, A1133-A1138. 
(18) Perdew, J. P.; Burke, K.; Ernzerhof, M. Generalized Gradient Approximation Made Simple. Phys. Rev. Lett. 1996, 77, 3865-3868.

(19) Fuchs, M.; Scheffler, M. Ab initio pseudopotentials for electronic structure calculations of poly-atomic systems using density-functional theory. Comp. Phys. Commun. 1999, $119,67-98$.

(20) Monkhorst, H. J.; Pack, J. D. Special points for Brillouin-zone integrations. Phys. Rev. B 1976, 13, 5188-5192.

(21) Onida, G.; Reining, L.; Rubio, A. Electronic excitations: density-functional versus many-body Green's-function approaches. Rev. Mod. Phys. 2002, 74, 601-659.

(22) Bechstedt, F.; Matthes, L.; Gori, P.; Pulci, O. Infrared absorbance of silicene and germanene. Appl. Phys. Lett. 2012, 100, 261906.

(23) Adolph, B.; Gavrilenko, V. I.; Tenelsen, K.; Bechstedt, F.; Del Sole, R. Nonlocality and many-body effects in the optical properties of semiconductors. Phys. Rev. B 1996, 53, 9797-9808.

(24) Yang, L.; Deslippe, J.; Park, C.-H.; Cohen, M. L.; Louie, S. G. Excitonic Effects on the Optical Response of Graphene and Bilayer Graphene. Phys. Rev. Lett. 2009, 103, 186802.

(25) Hogan, C.; Del Sole, R. Optical properties of the GaAs(001)-c(4 × 4) surface: direct analysis of the surface dielectric function. Phys. Stat. Sol. b 2005, 242, 3040-3046.

(26) Manghi, F.; Del Sole, R.; Selloni, A.; Molinari, E. Anisotropy of surface optical properties from first-principles calculations. Phys. Rev. B 1990, 41, 9935-9946.

(27) Giannozzi, P.; Baroni, S.; N. Bonini, M. C.; Car, R.; Cavazzoni, C.; Ceresoli, D.; Chiarotti, G. L.; Cococcioni, M.; Dabo, I.; Dal Corso, A. et al. QUANTUM ESPRESSO: 
a modular and open-source software project for quantum simulations of materials. $J$. Phys. Cond. Mat. 2009, 21, 395502.

(28) Giannozzi, P.; Andreussi, O.; Brumme, T.; Bunau, O.; Buongiorno Nardelli, M.; Calandra, M.; Car, R.; Cavazzoni, C.; Ceresoli, D.; Cococcioni, M. et al. Advanced capabilities for materials modelling with Quantum ESPRESSO. J. Phys. Cond. Mat. 2017, 29, 465901.

(29) Marini, A.; Hogan, C.; Gruening, M.; Varsano, D. Yambo: An ab initio tool for excited state calculations. Comp. Phys. Commun. 2009, 180, 1392-1403.

(30) Panzella, L.; Gentile, G.; D’Errico, G.; Vecchia, N. F. D.; Errico, M. E.; Napolitano, A.; Carfagna, C.; d'Ischia, M. Atypical Structural and $\pi$-Electron Features of a Melanin Polymer That Lead to Superior Free-Radical-Scavenging Properties. Angew. Chem. Int. Ed. 2013, 52, 12684-12687.

(31) Meng, S.; Kaxiras, E. Theoretical Models of Eumelanin Protomolecules and their Optical Properties. Biophys. J. 2008, 94, 2095-2105.

(32) Shioda, R.; van der Weide, J. Reflectance difference spectroscopy of highly oriented (2 $\times$ 1) reconstructed $\mathrm{Si}(001)$ surfaces. Phys. Rev. B 1998, 57, R6823(R).

(33) Palummo, M.; Onida, G.; Sole, R. D.; Mendoza, B. S. Ab initio optical properties of Si(100). Phys. Rev. B 1999, 60, 2522-2527.

(34) Caramella, L.; Hogan, C.; Onida, G.; Sole, R. D. High-resolution electron energy loss spectra of reconstructed $\operatorname{Si}(100)$ surfaces: First-principles study. Phys. Rev. B 2009, $79,155447$.

(35) Palummo, M.; Witkowski, N.; Pluchery, O.; Sole, R. D.; Borensztein, Y. Reflectanceanisotropy spectroscopy and surface differential reflectance spectra at the $\mathrm{Si}(100)$ surface: Combined experimental and theoretical study. Phys. Rev. B 2009, 79, 035327. 
(36) Cinquanta, E.; Fratesi, G.; dal Conte, S.; Grazianetti, C.; Scotognella, F.; Stagira, S.; Vozzi, C.; Onida, G.; Molle, A. Optical response and ultrafast carrier dynamics of the silicene-silver interface. Phys. Rev. B 2015, 92, 165427. 


\section{Graphical TOC Entry}

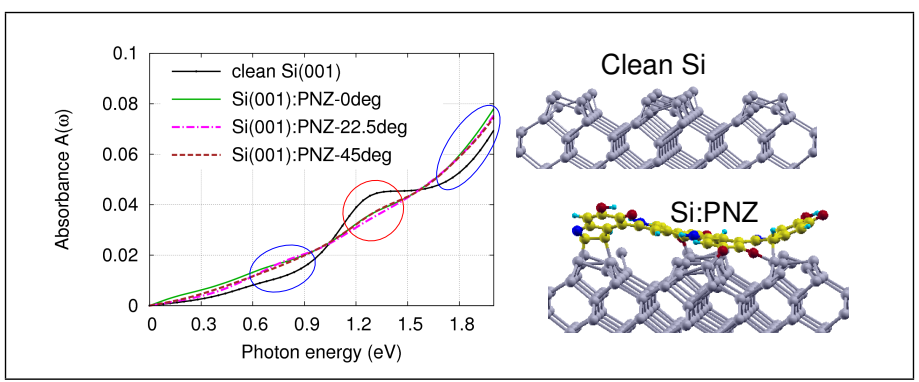

